\title{
Evolution of the EKA family of powdery mildew avirulence-effector genes from the ORF 1 of a LINE retrotransposon
}

\author{
Joelle Amselem ${ }^{1,2}$, Marielle Vigouroux ${ }^{3}$, Simone Oberhaensli ${ }^{4}$, James K. M. Brown ${ }^{3}$, Laurence V. Bindschedler ${ }^{5}$,
} Pari Skamnioti ${ }^{6}$, Thomas Wicker ${ }^{4}$, Pietro D. Spanu ${ }^{7}$, Hadi Quesneville ${ }^{1}$ and Soledad Sacristán ${ }^{8^{*}}$

\begin{abstract}
Background: The Avrk1 and Avra10 avirulence (AVR) genes encode effectors that increase the pathogenicity of the fungus Blumeria graminis f.sp. hordei (Bgh), the powdery mildew pathogen, in susceptible barley plants. In resistant barley, MLK1 and MLA10 resistance proteins recognize the presence of AVRK1 and AVRA10, eliciting the hypersensitive response typical of gene for gene interactions. Avrk1 and Avra10 have more than 1350 homologues in Bgh genome, forming the EKA (Effectors homologous to Avrk1 and Avra 10) gene family.

Results: We tested the hypothesis that the EKA family originated from degenerate copies of Class I LINE retrotransposons by analysing the EKA family in the genome of $\mathrm{Bgh}$ isolate $\mathrm{DH} 14$ with bioinformatic tools specially developed for the analysis of Transposable Elements (TE) in genomes. The Class I LINE retrotransposon copies homologous to Avrk1 and Avra10 represent $6.5 \%$ of the Bgh annotated genome and, among them, we identified 293 AVR/effector candidate genes. We also experimentally identified peptides that indicated the translation of several predicted proteins from EKA family members, which had higher relative abundance in haustoria than in hyphae.

Conclusions: Our analyses indicate that Avrk1 and Avra10 have evolved from part of the ORF1 gene of Class I LINE retrotransposons. The co-option of Avra 10 and Avrk1 as effectors from truncated copies of retrotransposons explains the huge number of homologues in Bgh genome that could act as dynamic reservoirs from which new effector genes may evolve. These data provide further evidence for recruitment of retrotransposons in the evolution of new biological functions.
\end{abstract}

Keywords: Barley, Powdery mildew, Blumeria graminis, Effector, Retrotransposon, LINE, Co-option

\section{Background}

Plant pathogens secrete effectors to influence host metabolism or defense mechanisms to provide an environment for successful infection [1]. Effectors can be recognized by plant resistance $(\mathrm{R})$ genes, eliciting effector-triggered immunity (ETI) in the plant to prevent further infection [2]. Effectors that are recognized by the plant are known as avirulence (AVR) proteins in the context of gene-for-gene interactions. Alteration of AVR genes enables parasites to

\footnotetext{
* Correspondence: soledad.sacristan@upm.es

${ }^{8}$ Centro de Biotecnología y Genómica de Plantas (UPM-INIA) and E.T.S.I. Agrónomos, Universidad Politécnica de Madrid, Campus de Montegancedo, Pozuelo de Alarcón 28223Madrid, Spain

Full list of author information is available at the end of the article
}

avoid R-dependent recognition and thus overcome host resistance [3].

The Avrk1 and Avra10 genes of the barley powdery mildew fungus, Blumeria graminis f.sp. hordei (Bgh), encode proteins which have a dual role as effectors and as AVR proteins, the presence of which is recognized by the MLK1 and MLA10 R-proteins. These genes were cloned from isolate CC148 (avirulent to barley plants carrying Mlk1 or Mla10 R-genes) by genetic and physical mapping [4]. Numerous lines of genetic and biological evidence indicate their dual function. The $A V R$ genes each co-segregate with the respective avirulence phenotype, have a functional open reading frame (ORF) in avirulent but not virulent isolates, and are expressed 
by isolates avirulent to the corresponding $R$-gene. The specific recognition of AVRK1 and AVRA10 by MLK1 and MLA10 R-proteins and subsequent cell death was shown in two independent series of experiments by transient expression of Avrk1 and Avra10 in barley plants carrying Mlk1 or Mla10 resistance genes $[4,5]$. Specific recognition of AVRK1 and AVRA10 also induced inaccessibility to subsequent infection and reduced fungal sporulation [4]. Their effector function was demonstrated in two independent experiments. First, when they were transiently overexpressed in susceptible plants lacking the corresponding $R$-gene, they increased the infectivity of Bgh [4]. Second, host-induced gene silencing (HIGS) of these genes in susceptible plants caused a reduction in haustorium formation by $B g h$ [5]. Further evidence for effector and AVR functions has been obtained for Avra10. This gene is expressed at low levels in conidia that have just landed on the leaf surface, followed by rapid induction within $6 \mathrm{~h}$ and high expression until $24 \mathrm{~h}$ after inoculation, with a drop thereafter [5]. The interaction between the MLA10 protein and the WRKY2 transcription factor depended on the presence of AVRA10 protein for defense gene activation [6]. An EMS-induced point mutation of Avra10, producing premature termination of the coding sequence, rendered the pathogen virulent to plants carrying the Mla10 resistance gene (i.e. avirulence was lost, so the Mla10 plant did not detect the fungus and induce effective defences) [4]. Natural Bgh isolates virulent to Mla10 or Mlk1 do not have functional variants of Avrk1 or Avra10 in the corresponding loci, in many cases due to the fusion of the $A V R$ genes with retrotransposon sequences [4].

Avrk1 and Avra10 are homologues (with $64 \%$ nucleotide and $43 \%$ protein identities), and are the first discovered members of the EKA gene family (Effectors homologous to Avrk1 and Avra 10), with more than 1350 homologues in the $B g h$ genome [7]. The EKA family has no known homologues outside powdery mildew fungi [8] but is present in different formae speciales of Blumeria graminis [9] and in powdery mildew species infecting different host such as pea, grape, plantain or Arabidopsis thaliana [9-11]. A previous analysis showed that these Avrk1 and Avra10 homologues are frequently found in the same ORF as the nucleotide binding (NB) domain of a LINE retrotransposon, that the AVR-homologous and NB domains are expressed in different $B g h$ isolates as a single transcript, and that both type of sequences have coevolved [9].

LINE retrotransposons are almost ubiquitous in fungi, plants and animals [12]. They are able to replicate autonomously, and their mobility is dependent on target-primed reverse transcription [13]. These elements typically consist of an ORF (usually called ORF2) containing a gene encoding a reverse transcriptase (RT) and an endonuclease. In addition, ORF1, usually found in the $L 1, I$ and Jockey groups $[12,14]$, may have been acquired independently on multiple occasions during the evolution of LINE elements [15]. The best studied ORF1 protein (ORF1p) is that in the $L 1$ superfamily of LINE elements. ORF1p is thought to assist $L 1$ retrovirus-like particles to gain access to the nucleus, where it can interact with genomic DNA and thus initiate integration through target primed reverse transcription [16]. The ORF1p of the second major superfamily of LINEs (named " $P$ ") is only poorly characterized and its function is not yet fully understood [17]. It usually contains at least one non-canonical RNA-recognition motif (RRM) domain and one or more $\mathrm{CCHC}$ zinc knuckle motifs [18]. ORF1 proteins evolve much faster than RT proteins, leading to them being very poorly conserved between lineages. That means that RT proteins from distantly related species still show strong homology, while ORF1 proteins have virtually no similarity [18].

In the work reported here, the EKA family in the genome of Bgh isolate DH14 (BluGen, Blumeria Genome Sequencing Consortium, http://www.blugen.org/, [7]) has been analysed with pipelines from the REPET package, which allows de novo detection, classification and annotation of transposable elements (TEs) in whole genomes [19-21]. This method detects TE sequences in the genome and groups them according to a putative common ancestor represented by a consensus sequence (called a TE consensus). The method then identifies the matches between each $\mathrm{TE}$ consensus and the genomic sequence (TE fragments) and reconstructs the TE copies in the genome, even if they are nested and degenerated. A TE copy is a chain of matches between each TE consensus and the genomic sequence, each match in the chain being a TE fragment. Hence, a full-length TE copy may correspond to several TE fragments, which, when connected together, correspond to the full TE consensus sequence [19].

Our results show that Avrk1 and Avra10 have evolved from part of the ORF1 gene of Class I LINE retrotransposons, which we have named Kryze and Satine, respectively. The activity of the ancestors of these elements generated a high diversity of degenerate copies. This provides a possible mechanism for the extensive proliferation of the $E K A$ family in the $B g h$ genome. These results imply that Avrk1 and Avra10 originated from the truncated ORF1 of Class I-LINE retrotransposons, in a recycling and neofunctionalization process in which the retrotransposon genes were recruited by the Bgh genome as effectors. The barley plant then evolved to recognize the presence of the $B g h$ parasite through the presence of these retrotransposonderived effector genes in the fungal genome.

\section{Results}

\section{Avrk1 and Avra10 are homologues of the ORF1 of LINE 1 retrotransposons}

Our previous results indicated that the $E K A$ gene family was very large and related to TEs. Thus, we based the 
analysis of the evolution of this family on identification and classification of TE consensus sequences with homology to Avrk1 and Avra10. A first step made use of Bgt_RIX_Inari, a complete TE consensus characterized in B. graminis f.sp. tritici (Bgt) [22] that had been classified as a LINE retrotransposon and contained a region highly similar to Avra10. We identified several fragments homologous to Bgt_RIX_Inari in the genome of Blumeria graminis f.sp. hordei $(B g h)$ isolate DH14 that were then used to build manually a consensus model for Bgh that we called Satine, which represents a LINE retrotransposon with two ORFs (Fig. 1). ORF1 is homologous to Avra10 (99 \% nucleotide identity, Additional file 1: Figure S1) and contains, in the 3 ' region, a sequence coding for a cysteine-rich NB domain conserved between different TEs $[9,23]$. ORF2 is homologous (51 \% of amino acid identity) to the reverse transcriptase and RNase H (RT$\mathrm{RH}$ ) of the retrotransposon CgT1 identified in the fungal plant parasite Glomerella cingulata (anamorph Colletotrichum gloesporioides; Fig. 1; [23]).

We then refined the TE annotation of the Bgh genome using the TEdenovo pipeline and several rounds of the TEannot pipeline. Satine and two previously characterized Bgh class I SINE retro-elements (Bgh_EGR1_cons and Bgh_EGH24_cons) [24, 25] were added to the Repbase library [26] used by the TEdenovo pipeline to classify TE consensus sequences. We finally obtained a library of 733 TE consensus sequences (hereafter named Blgr_refTEs). TE annotation with Blgr_refTEs accounted for $67.1 \%$ of $B g h$ genome. Class I LINE and LTR retrotransposons were the most abundant TEs in Bgh genome, accounting for $24.5 \%$ and $21.7 \%$ of Bgh genome assembly, respectively (Table 1).

In order to identify the TE consensus sequences most similar to Avra10 and Avrk1 in the Bgh_refTEs library, we performed a blastx-based sequence comparison [27]. We recovered 13 and 9 consensus sequences highly similar (e-value < 1e-15) to Avra10 and Avrk1 respectively and $14 \mathrm{TE}$ consensus sequences similar to both Avra10 and Avrk1 (Table 2). These consensus sequences contain 7513 genomic copies and represent $5.68 \mathrm{Mbp}(6.5 \%)$ of the $B g h$ annotated genome ( $88 \mathrm{Mbp})$. Two TE consensus sequences (Bgh_RIX_G5642 and Bgh_RIX_G5682), classified by REPET as LINE retrotransposons, span the whole Satine consensus model (Fig. 2). These three consensus sequences (Satine, Bgh_RIX_G5642 and Bgh_RIX_G5682), were used to annotate four, one and two Satine-like full-length copies respectively, in the genome. The plot of the location of the genome copies on their respective TE consensus sequences shows that they are smoothly scattered along the whole consensus model, with no break points indicative of chimerization events (Fig. 2). The absence of break points indicates that Avra10 is part of the original ORF1 of the Satine Class I-LINE retrotransposon.

Any of the nine TE consensus sequences similar to Avrk1 could be considered full-length LINE retrotransposons containing both ORF 1 and ORF 2. Seven out these nine TE consensus sequences matched 34 full-length fragments spanning the whole TE consensus sequence. In order to look for putative ORF2 that had been overlooked during TE search and annotation, we extracted the genome sequences of these 34 fragments and searched for LINE-ORF2 domains within the $3000 \mathrm{bp}$ downstream of the ORF1. PASTEC TEclassifier [20] identified the characteristic RT-RH domain at the expected location in one of those sequences (Fig. 3A). Thus, we found a potential genome copy of a full-length LINE retrotransposon with 2 ORFs (that we hereafter name Kryze) in a full-length genome copy annotated by TE consensus Bgh_RIX_G4472 ( $81.5 \%$ of nucleotide identity), extended to 3000 bp downstream. The ORF 1 of Kryze is similar to Avrk1 (67\% amino-acid identity), and the extended $3000 \mathrm{bp}$ containing the ORF2 of Kryze are similar (71.5\% nucleotide identity) to the TE consensus Bgh_RIX_G5646 (Fig. 3B).

\section{Characteristics of the EKA family}

The alignment of AVRK1 and AVRA10 with the ORF1p of the respective elements (Kryze and Satine) indicates that both proteins have lost the $\mathrm{C}$-terminal half that contains the conserved NB domain typical of an ORF1p (Figs. 2 and 3). Hence, candidates for other AVR/effector genes may consist of truncated copies of the ORF1p without the region containing the $\mathrm{NB}$ domain, thus spanning only the region homologous to AVRK1 or AVRA10. We

Fig. $\mathbf{1}$ Consensus model Satine, which represents a LINE retrotransposon with two ORFs. ORF1 is homologous to Avra10 (99 \% nucleotide identity
of Avra10 to the corresponding portion of ORF1) and contains, in the 3' region, a sequence coding for a cysteine-rich nucleotide binding domain
conserved between different TEs (NB). ORF2 is homologous to the reverse transcriptase and RNase H (RT-RH) of the retrotransposon CgT1 identified in
the fungal plant parasite Glomerella cingulata. UTR: Untranslated region


Table 1 Classification, number, content and genome coverage of TE consensus sequences annotated in Bgh genome.

\begin{tabular}{llll}
\hline Class, Order & $\begin{array}{l}\text { Number of consensus } \\
\text { sequences }\end{array}$ & TE content (\%) & $\begin{array}{l}\text { Genome } \\
\text { coverage (\%) }\end{array}$ \\
\hline Class I, LTR & 358 & 32.3 & 21.7 \\
Class I, LINE & 241 & 36.5 & 24.5 \\
Class I, SINE & 20 & 16.4 & 11.0 \\
Class II, MITE & 6 & 0.7 & 0.5 \\
Class II, TIR & 7 & 1.1 & 0.7 \\
Unclassified & 101 & 13.0 & 8.7 \\
Total & 733 & 100 & 67.1 \\
\hline
\end{tabular}

extracted the 7513 genomic copies homologous to Avrk1 and Avra10 and searched for the sequences spanning either the full length or the truncated ORF1p (these last being considered as AVR/effector candidates). We found 135 AVR/effector candidates homologous to AVRK1, 68 AVR/effector candidates homologous to AVRA10, and 90 AVR/effector candidates equidistant from both AVRAK1 and AVRA10 (Table 3). The mean identity among truncated sequences was lower than the identity between the sequences homologous to the full-length ORF1p for all three types of sequences (Table 3). Also, the redundancy was eight times higher in full-length ORF1 sequences than in truncated sequences (3/123 versus $1 / 294$ redundant sequences, Table 3 ). Hence, truncated sequences are less conserved than full-length ORF1 sequences.

Phylogenetic analysis classifies the sequences from Bgh isolate DH14 homologous to AVRK1 or AVRA10 in two clades (Fig. 4). Clade 1 contains mainly AVRK1 homologues and a few equidistant sequences and groups them with the Kryze ORF1p and with AVRK1 from isolate CC148. Clade 2 groups all the remaining sequences in five subclades. Subclade 2A1 contains only AVRA10 homologues and groups them with Satine ORF1p and with AVRA10 from isolate CC148. The phylogeny indicates that stop codons between the AVR-homologous sequence and the NB domain have appeared several times during the evolution of the EKA family, since the truncated sequences appear in the same clades as the full length ORF1 sequences (Fig. 4). We tested if truncated sequences were subject to different selection patterns compared to the full-length sequences, indicative of different functions. We analysed the non-synonymous/ synonymous substitution rate ratio $(\mathrm{dN} / \mathrm{dS}=\omega)$ to detect positive selection $(\omega>1)$ in the full length and truncated sequences homologous to AVRK1 or AVRA10 (Fig. 5). We found evidence of positive selection with variable selective pressure among sites in the four groups of sequences analyzed $(P<0.01)$ (AVRK1-like full length, AVRK1-like truncated, AVRA10-like full length and AVRA10-like truncated). There were different positively selected sites in the AVRK1-like truncated sequences than in the full-length TE ORF1p: sites 125P, 127S, 149I and $172 \mathrm{H}$ are positively selected in AVRK1-like truncated sequences and sites 233 L, 294I, 298Y and 316 L, situated after the NB domain, are positively selected in the sequences corresponding to full-length TE ORF1p (Fig. 5). For AVRA10-like sequences, the Likelihood Ratio Test (LRT) did not identify significant positively selected sites either in the truncated sequences or in the full-length TE.

We searched for specific domains in ORF1 predicted proteins in both full length and truncated sequences. There was no similarity with any known ORF1 protein. There was weakly significant similarity $\left(E\right.$-value $<\mathrm{e}^{-03}$ ) to retrovirus zinc finger-like domains in both full-length and truncated sequences, whilst predicted coiled coils were only found in full-length ORF1ps.

\section{EKA protein identification}

Several predicted EKA proteins were identified experimentally in infected epidermis (haustoria samples) and in sporulating hyphae from 5-7 dpi infected barley plants (Table 4 and Additional file 2: Table S1). Most peptides contained proline residues, as they were identified from sequences with $>8 \%$ proline content and many were detected as hydroxyprolines. The presence of proline is detrimental for mass spectrometry peptide fragmentation using collision induced dissociation (CID) as the "proline effect" leads to a poor fragmentation pattern [28]. This may explain the low Mascot peptide scores, just above the identity threshold, for most detected peptides which contain proline-rich residues. Since proteins could only be identified with two or three peptides, it was not possible to discriminate well between isoforms of closely related proteins, hampering determination of the exact number of proteins in each sample (Additional file 3: Table S2). However, it is clear that several EKA proteins are differentially

Table 2 Metrics of the LINE consensus sequences homologous to AVRK1 or AVRA10

\begin{tabular}{|c|c|c|c|c|c|c|c|}
\hline TE & $\begin{array}{l}\text { Number of consensus } \\
\text { sequences }\end{array}$ & $\begin{array}{l}\text { Consensus } \\
\text { max length (bp) }\end{array}$ & Genome coverage (Mb) & $\begin{array}{l}\text { Number of } \\
\text { fragments }\end{array}$ & $\begin{array}{l}\text { Number of full } \\
\text { length fragments }\end{array}$ & $\begin{array}{l}\text { Number of } \\
\text { copies }\end{array}$ & $\begin{array}{l}\text { Number of full } \\
\text { length copies }\end{array}$ \\
\hline LINE/AVRK1 & 9 & 4468 & 1.43 & 2370 & 46 & 2370 & 74 \\
\hline LINE/AVRA10 & 13 & 6014 & 1.56 & 2040 & 18 & 1566 & 37 \\
\hline $\begin{array}{l}\text { LINE similar to } \\
\text { bothAVRK1/AVRA10 }\end{array}$ & 14 & 6083 & 2.69 & 3577 & 39 & 3577 & 77 \\
\hline Total & 36 & 6083 & 5.68 & 7987 & 103 & 7513 & 188 \\
\hline
\end{tabular}



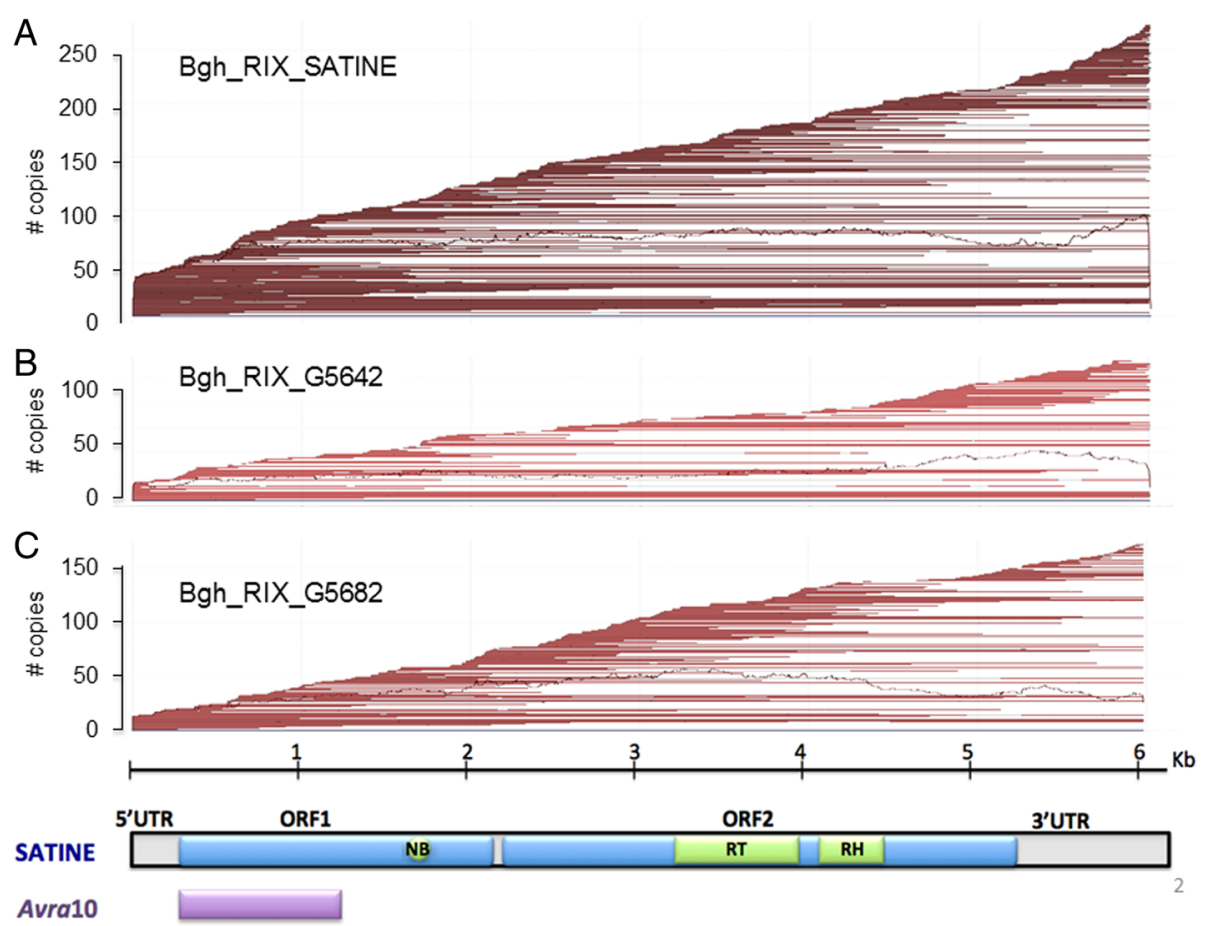

Fig. 2 Genome TE copies plotted on the consensus sequences Satine (a), Satine-like Bgh_RIX_G5642 (b) and Satine-like Bgh_RIX_G5622 (c). Brown lines represent the part of the consensus sequence that aligns with each annotated copy. Copies are ordered according to their coordinates (Start, End). The black curve represents the depth of coverage along the reference sequence

expressed in various fungal tissues, since most protein hits were identified only in either the haustoria or the hyphae (15 and 14 proteins respectively), with only four proteins identified in both types of sample (Table 4). The possible 33 validated proteins were identified from 15 detected peptides in the haustoria samples and 21 peptides in the hyphae samples. There was almost no overlap of identified peptides between tissues; only the peptide AAAPLPLR was identified in both the haustorial and hyphal samples (Table 4). Most of the identified EKA proteins were AVRA10-like, notably those in haustorial samples (Table 4), with most of the hits in subclade 2A1 that contains Satine ORF1p (Fig. 4), including a copy of the TE consensus Bgh_RIX_ G5682 that spans the whole Satine consensus model. A copy of consensus Bgh_RIX_G5642, which also spans the whole Satine consensus model, was found in a hyphal sample. AVRK1-like proteins were only found in hyphal samples (Table 4). Thirteen of the 33 identified proteins are truncated ORF1s and are thus putative AVR/effectors. Most of them (11) are AVRA10-like and appear in haustorial samples, whereas only one truncated protein is AVRK1-like (Table 4). Additional AVRA-10 proteins in the subclade $2 \mathrm{~A} 1$ were identified in haustoria with one significant peptide and a peptide just below the identity score threshold (Fig. 4 and Additional file 3: Table S2), reinforcing the hypotheses that some haustorium-specific AVRA10 effectors are expressed as proteins.

\section{Discussion}

The genomes of powdery mildew fungi are amongst the largest of ascomycete fungi, due to an extraordinary proliferation of TEs $[7,29]$. This is probably, in part, the result of the absence of the RIP (Repeat-induced point mutation) pathway to control genetic parasites that is otherwise conserved in all related ascomycetes [7, 30]. TE proliferation and genome expansion may have had deleterious effects on powdery mildews, entailing a considerable loss of genes that has resulted in these pathogens being entirely dependent on living host cells [7]. However, TE activity has also benefited powdery mildews and other eukaryotic pathogens, favoring the expansion and diversification of a broad repertoire of effector genes, as previously shown [31-33]. Here, we show an additional way in which transposition activity contributed to the evolution of B. graminis, by the recycling and neofunctionalization of degenerate TE products which may generate new effector genes. The products of at least two of these genes, Avrk1 and Avra10 are recognized by the host plant as avirulence proteins and it is conceivable that other members of the $E K A$ family may encode avirulence genes.

Our results indicate that the origin of Avrk1 and Avra10 is the truncated ORF1 of the retrotransposons Satine and Kryze respectively (Figs. 2 and 3). Satine and Kryze are members of the $I$ superfamily of LINEs, 


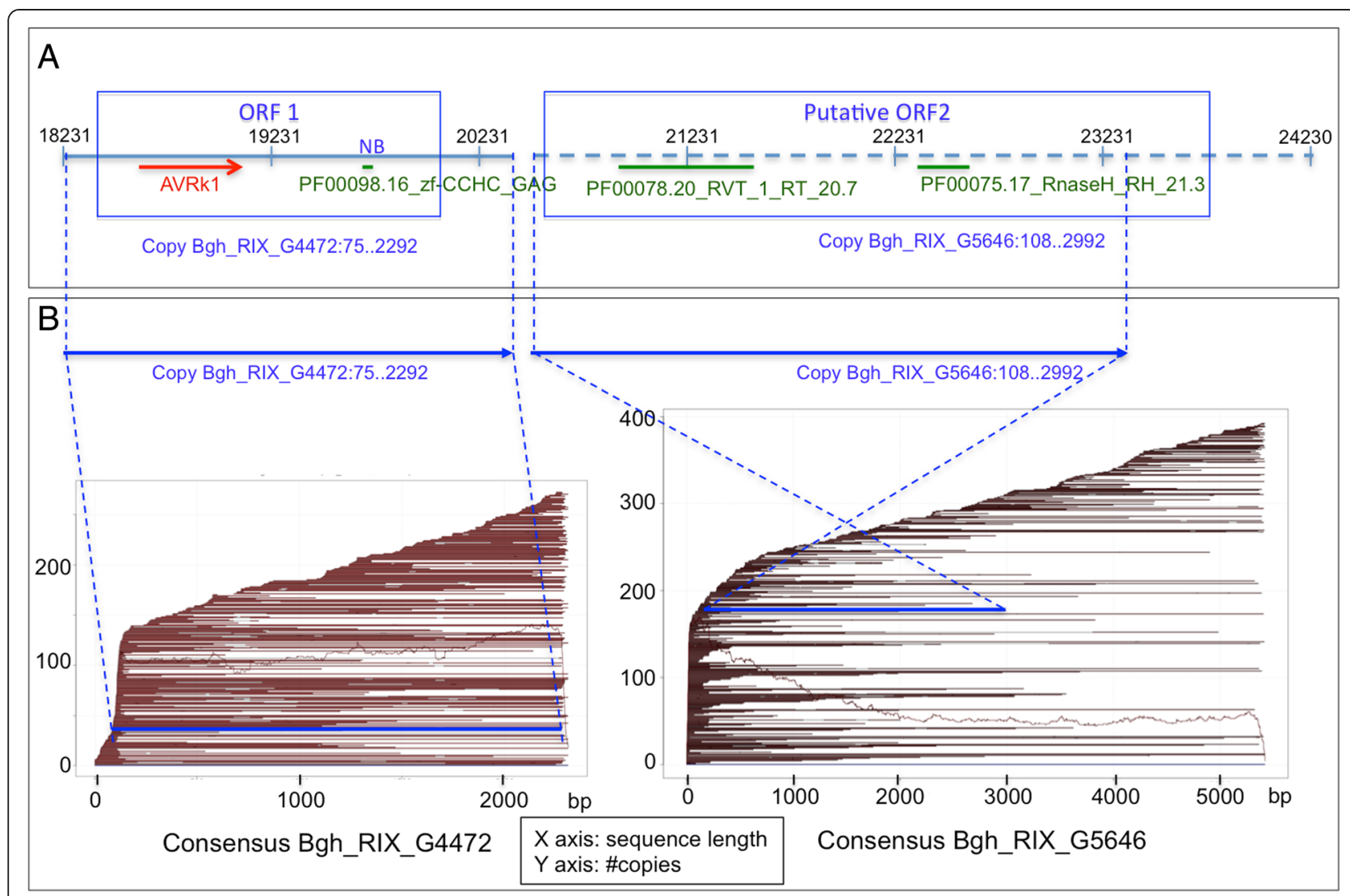

Fig. 3 Kryze potential genome copy containing an ORF1 homologous to AVRk1 containing a cysteine-rich NB domain (PF00098.16_zf-CCHC_GAG) and a putative ORF2p with RT (PFAM: PF00078.20) and RH (PFAM: PF00075.17) domains. a Blgr_V3_contig_001018.fa:18231..24230 bp corresponding to Bgh_RIX_G4472 full-length copy with 3000 bp downstream region. AVRK1 alignment (in red) and domains annotated by PASTEC classifier (green) are represented. $\mathbf{b}$ Blue lines represent the two TE genomic copies mapped in the context of all the copies (brown lines) plotted to the reference TE consensus sequences Bgh_RIX_G4472 and Bgh_RIX_G5646

containing protein domains typical of that superfamily and showing characteristic sequence organization. The $I$ superfamily is the only group of LINEs that contains a RNAseH domain in their ORF2, together with the Randl group and some members of the $L 1$ superfamily. Additionally, the $I$ superfamily is characterized by having an ORF1 upstream of the ORF2, with zinc finger-like domains $[12,14]$. Our structure analysis showed that the ORF1 of Satine and Kryze contains a zinc finger-like domain characteristic of ORF1 of the LINE I elements. Because ORF1 proteins are known to evolve very rapidly, it is not surprising that no homologues of Satine and Kryze elements were found outside the powdery mildew fungi [8].

The expansion of Satine and Kryze lineages may have contributed to the expansion of other protein effectors of the EKA family. Avrk1 and Avra10 were isolated from $B g h$ isolate CC148, which is avirulent to barley plants

Table 3 Number of sequences per group (non-redundant sequences between parenthesis) and mean identity percentage in each group for full length ORF1 and truncated sequences homologous to AVRK1 or AVRA10.

\begin{tabular}{llllll}
\hline & \multicolumn{2}{l}{ Full length ORF1 sequences } & & \multicolumn{2}{l}{ Truncated sequences before NB domain (putative AVR/effectors) } \\
\cline { 2 - 3 } & Number of sequences & Mean identity & & Number of sequences & Mean identity \\
\hline AVRK1-like & $30(28)$ & $69.3 \%$ & $135(135)$ & $57.0 \%$ \\
AVRA10-like & $32(32)$ & $59.9 \%$ & $69(68)$ & $55.2 \%$ \\
Similar to bothAVRK1/AVRA10 & $61(60)$ & $58.3 \%$ & $90(90)$ & $49.2 \%$ \\
Total & $123(120)$ & & $294(293)$ &
\end{tabular}

${ }^{a}$ Mean identity percentage in each group is based on translated full-length ORF calculated by using the pairwise identity after realigning each pair of sequences and averaging it out for all sequences in the group. 


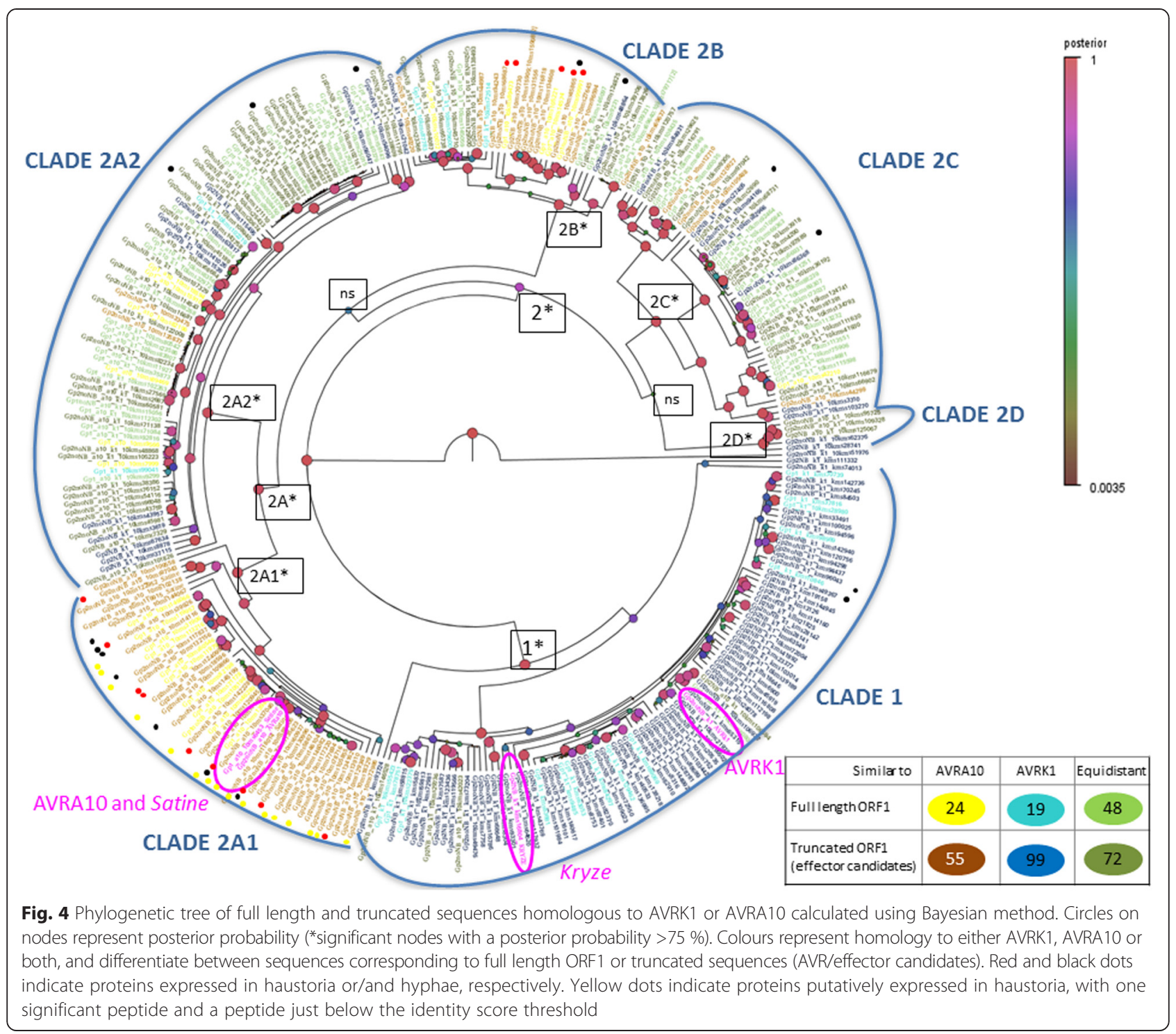

carrying resistance genes Mla10 and Mlk1 [4]. Isolate DH14 is virulent to barley plants carrying Mla10 and Mlk1 and does not contain functional variants of Avrk1 or Avra10 in the corresponding loci delimited by genetic and physical mapping [4]. However, this and previous works have found $E K A$ homologues in DH14 and other $B g h$ isolates, many of which are transcribed [7-9]. Thus, these homologues may function as effectors in these other isolates and also be $A V R$ genes recognized by $R$ genes other than Mla10 and Mlk1. We have found 293 truncated copies of Satine and Kryze ORF1ps that may be AVR/effector candidates because they span the region homologous to Avrk1 or Avra10 and lack the downstream sequence containing the NB domain (Table 3 ). The lower degree of conservation of truncated sequences compared to full-length ORF1 sequences (Table 3) could be due to diversifying selection concomitant with the expansive generation of effectors. Different positively selected sites were found in the AVRK1-like truncated sequences than in the full-length TE ORF1p (Fig. 5), which suggests that the two groups of sequences have different functions. However, the likelihood ratio test did not find significant positively selected sites in AVRA10 homologues. This could be due to a lower number of analyzed AVRA10 homologues and/or to the fact that AVRA10 homologues are phylogenetically very heterogeneous, in contrast to AVRK1 homologues, which are mostly grouped in a single clade that also contains the Kryze ORF1 (Fig. 4). A previous analysis did not find Avrk1 homologues in B. graminis parasitic on oats or ryegrass (Lolium perenne), while Avra10 homologues are present in the genomes of forms of $B$. graminis parasitic on oats, ryegrass, barley and the closely related species rye, wheat and Elymus repens [9]. This implies that the 


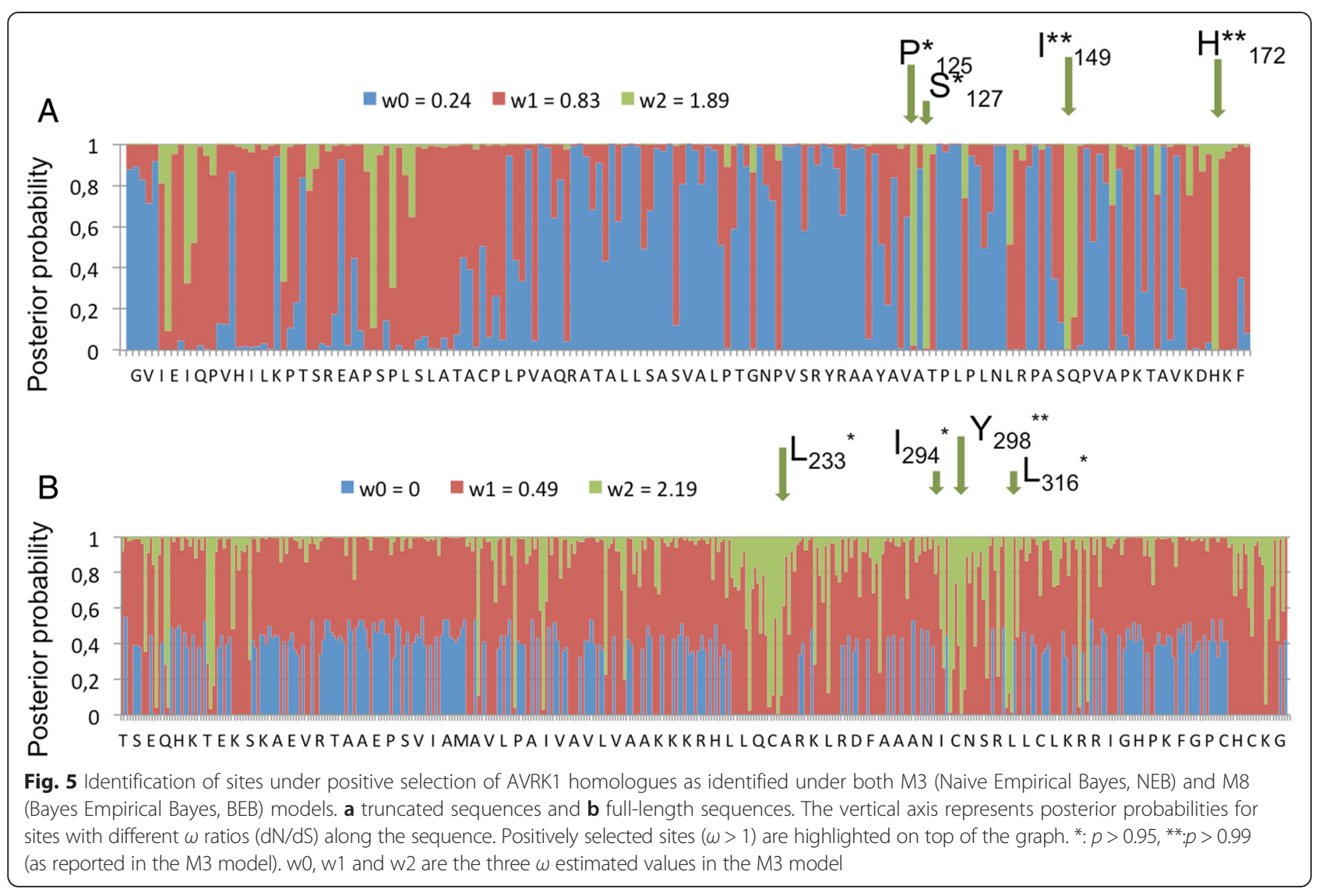

$E K A$ family proliferated during the evolution of different forms of B. graminis, but the Avrk1 clade (or Kryze lineage) only proliferated after the divergence of B. graminis on oat and ryegrass from the other forms [34]. Hence, different $E K A$ lineages could be related to the adaptation of $B$. graminis to different hosts.

Our results suggest that there has been a continuous process of recruitment and neofunctionalization of truncated ORF1 copies. Diversification and amplification of this family of effector genes most likely happened as a result of strong natural selection by the host. Loss or mutation of an AVR/effector protein would have resulted in the pathogen not being recognized by the plant host but would have generated selection pressure for its replacement by other effector proteins (Fig. 6). The concept of neofunctionalization and co-option of TE sequences by host genomes was proposed by Brosius and Gould [35], who suggested the term "exaptation" for the phenomenon of "junk" DNA sequences such as TEs acquiring a novel function in the genome. More recently, genome-scale analyses have confirmed that domesticated or exapted TE-derived sequences have contributed diverse and abundant regulatory and protein-coding sequences to host genomes [36]. The new functions which arose from recruitment of TE sequences range from their capture as cisregulatory sequences at promoter and enhancer regions
[37] to the most extensive gene domestication in which single copy genes are well conserved among lineages [38]. Examples of TE domestication are the element of the transib superfamily from which the V(D)J system at the base of the vertebrate immune system evolved [39], the HERVderived Syncitin genes that play crucial roles in placenta formation [40] and the non-LTR TE origin of telomeres and telomerases $[41,42]$. The evolution of the $E K A$ family of effectors in a plant pathogenic fungus further indicates the extraordinary diversity of functions which have evolved from retroelements.

AVRK1 and AVRA10 have been shown to function as effectors because they enhance $B g h$ infectivity $[4,5]$. It is predicted that $A V R$ genes recognized by plant $R$ genes should confer a selective advantage if they are to be retained in the pathogen population (reviewed in [43]). While AVRK1 and AVRA10 have effector activities as well as being recognized as avirulence proteins [4-6], their precise functions in the interaction of $\mathrm{Bgh}$ with its host are unknown. There are several examples that show the transcription of $A V R K 1$ and $A V R A 10$ and other members of the $E K A$ family [4, 9-11]. Here, we provide the first experimental evidence indicating the production of EKA proteins by Bgh. We identified 15 proteins putatively expressed in the haustorial samples and 14 proteins putatively expressed in the hyphal samples (Table 4). This 
Table 4 Proteomic analysis of haustoria and hyphae samples for experimental identification of EKA proteins.

\begin{tabular}{|c|c|c|c|}
\hline & Haustoria only & Hyphae only & Haustoria and hyphae \\
\hline \multicolumn{4}{|l|}{ Proteins identified } \\
\hline \multicolumn{4}{|l|}{ AVRK1-like } \\
\hline Full length ORF1 & 0 & 3 & 0 \\
\hline Truncated (putative AVR/effectors) & 0 & 1 & 0 \\
\hline Total & 0 & 4 & 0 \\
\hline \multicolumn{4}{|l|}{ AVRA10-like } \\
\hline Full length ORF1 & 6 & 5 & 1 \\
\hline Truncated (putative AVR/effectors) & 8 & 0 & 3 \\
\hline Total & 14 & 5 & 4 \\
\hline \multicolumn{4}{|l|}{ Similar to both AVRK1/AVRA10 } \\
\hline Full length ORF1 & 1 & 4 & 0 \\
\hline Truncated (putative AVR/effectors) & 0 & 1 & 0 \\
\hline Total & 1 & 5 & 0 \\
\hline Total proteins identified & 15 & 14 & 4 \\
\hline \multirow[t]{22}{*}{ Total peptides } & 15 & 21 & 1 \\
\hline & ATVPALPR & AGLGRSAGPSITK & AAAPLPLR \\
\hline & DDRIFLR ${ }^{a}$ & AHFHPSTR & \\
\hline & GLESNIQNMNAIAAALLAKENDVPEVDMVDAEVEK & APKKEAPK & \\
\hline & KASGPAETSR & ARPSKSGPVK & \\
\hline & KPTPPTKKSPK & ASDLVALR & \\
\hline & MLELSQTK & ASIAQFIQAGPGATPPVLPK & \\
\hline & MPTPPTKQSAK & DQLVTIR & \\
\hline & MPTPPTKR & EKTIEPAENSTRK & \\
\hline & NAVSGTAKNR & ELLDSSTSRSVSGIK & \\
\hline & NMPEVDMVDAEVEKLK & EPPNQPTPVMVSRAPK & \\
\hline & RATVPALPR & FLPSLPQRQATQGK & \\
\hline & SPKTATLSSPDNR & KMESDMTER & \\
\hline & SWAALFPR & KREELLDAAPK & \\
\hline & TLSPFGGR & LFQMPPTR & \\
\hline & VPTSFALR & MPTPPSKKLAK & \\
\hline & & QGIPPGSIER & \\
\hline & & SDSRSCKSRPNK & \\
\hline & & SKKSGPVTK & \\
\hline & & SLAHKAAETDK & \\
\hline & & SWAALFPRNASPKPGFR & \\
\hline & & VPTGFTLR & \\
\hline
\end{tabular}

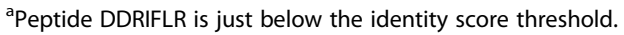

makes a ratio of 1:1 for proteins identified in the haustoria versus the hyphae that, despite the difficulty to determine the exact number of proteins actually expressed, is clearly higher than the ratio found in a global proteomic analysis of these tissues, which is 1:4.5 [44]. Also, 15 and 21 different peptide sequences were identified in haustorial and hyphal samples respectively. Together, these data indicate that the EKA proteins may be more abundant and diverse in haustoria than in sporulating hyphae. This is consistent with the EKA proteins accumulating or being secreted in association with haustorial structures like other fungal effectors [45], although the secretion mechanism is unknown. The identified peptides associated with EKA proteins are almost exclusive to either haustoria or hyphae, with only one peptide shared by both types of samples (Table 4), implying that different EKA proteins may have 


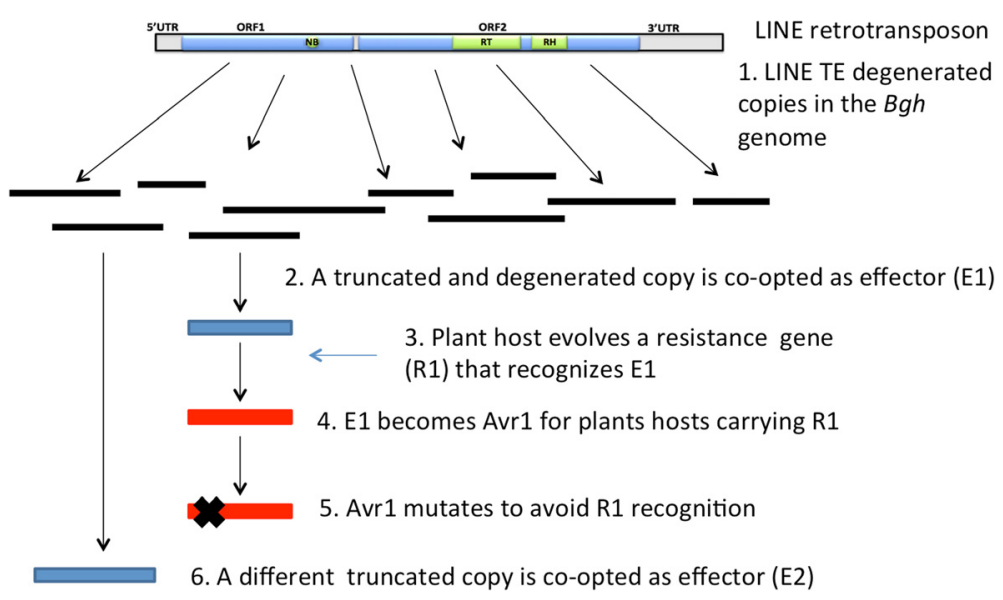

Fig. 6 Model for the evolution of the EKA family by co-option as effectors by Bgh genome. 1. Genome rearrangements after retrotransposon activity produce degenerate copies of LINE retrotransposons in the Bgh genome. Some of these copies are truncated ORF1s. 2. One truncated ORF1 is co-opted as effector E1 that enhances pathogenicity. 3. Plant host evolves a resistance gene R1 that recognizes the presence of E1. 4. E1 becomes an avirulence gene (Avr1) if it is recognized by R1 in a gene-for-gene interaction. 5. Avr1 mutates to avoid recognition by R1. 6. Bgh co-opts other degenerate copies as effectors that contribute to enhanced pathogenicity if they are not recognized by host $R$ genes. During this process, Bgh genome and EKA copies are evolving, and the copy co-opted at point 6 may not have existed at point 2 and could have come from a different EKA ancestor

specific functions in different fungal tissues. These functions may also be specific to the EKA protein subfamily, since most of the haustorium-associated proteins are AVRA10-like but none of the AVRK1-like proteins were found in haustoria. Most of the AVRA10-like haustoriumassociated proteins were truncated, unlike AVRK1-like proteins, which were mainly full length and were thus probably functional LINE ORF1s. The functions of the EKA proteins could be related to their biochemical or structural properties. On the biochemical side, they are proline-rich. One example of a fungal proline-rich protein $(\mathrm{C} 1 \mathrm{H} 1)$ is expressed exclusively in plants and has a role in biotrophy during Colletotrichum-host interactions [46, 47]. This gene has orthologs in C. higginsianum and Passalora fulva (anamorph Cladosporium fulvum) that are also proline-rich and are involved in chitin sequestration and camouflage to avoid plant recognition [48, 49]. On the structural side, the ORF1p of superfamily $I$ of LINE TEs, from which the EKA family derives, contains noncanonical RRM domains through its whole length [18]. AVRK1 and AVRA10 and other EKA members may have conserved part of those domains. Both truncated and full length ORF1 predicted proteins show weak similarities with zinc finger-like domains typical of retroviral Gag-proteins. These domains are involved in protein interactions with RNA, DNA or other proteins [50]. Any of the properties of these domains could have been retained to give a novel function to AVRK1, AVRA10 and other related AVR/effector candidates and thus a selective advantage to Bgh individuals carrying them.

Functional innovation by exaptation has been related to extremely stressful situations or crises such as the mass extinction 250 Mya that gave rise to mammals [51]. There are several examples of increased TE activity associated with stress in insects and plants [36]. Indeed, TE mediated genetic variation can be fundamental in the host genome's evolutionary response to stress, facilitating the adaptation of populations and species to changing environments [52]. The role of TEs in parasite-host coevolution as mutators which increase parasite genomic plasticity has been discussed $[32,53]$. Recently, retrotransposons have been shown to increase pathogenesis and virulence by silencing either pathogen avirulence genes or plant defense mechanisms $[54,55]$. Here, we provide evidence of a direct role of retrotransposons in virulence as dynamic reservoirs from which new effector genes evolve. It is especially striking that the host plant recognizes the presence of the powdery mildew parasite by the presence of genes which evolved from a genomic parasite - a retrotransposon family - of the fungus.

\section{Conclusions}

Transposable elements account for $67 \%$ of Bgh genome size, and Class I-LINE retrotransposons are the most abundant in the Bgh genome, representing $24.5 \%$ of genome coverage and $36.5 \%$ of the TE content (Table 1). The sequences homologous to Avrk1 and Avra10 (the EKA family) within the repetitive DNA landscape of the $B g h$ genome have been identified and analysed, and the origin of these AVR/effector genes has been determined to be the truncated ORF1 of the LINE retrotransposons Satine and Kryze (Figs. 2 and 3). Thus, Avrk1 and Avra10 genes 
have been co-opted in the Bgh genome as effectors from truncated copies of retrotransposons.

DNA repeats of Satine-like and Kryze-like lineages represent at least $6.5 \%$ of the genome assembly (Table 2). These data indicate a long-standing activity of these two elements in Bgh genome, during which a high diversity of degenerate copies has been generated. New effector genes may have evolved from these degenerated copies in a recycling and neofunctionalization process driven by the coevolution between Bgh and its host plant (Fig. 6). We have found 293 truncated copies of Satine and Kryze ORF1s that may be AVR/effector candidates (Table 3) and peptides that indicate translation of these genes (Table 4). These candidates have arisen several times during the evolution of the EKA family (Fig. 4), and belong to different lineages that could be related with the adaptation of $B$. graminis to different hosts.

\section{Methods}

\section{Satine consensus manually built}

Bgt_RIX_Inari, a LINE element detected in Bgt-Repeat Library [22], is highly similar to Avra10, an AVR/effector gene from Bgh isolate CC148 [4]. Homologues of Bgt_RIX_ Inari were identified in the genome of $B g h$ isolate DH14 (BluGen, Blumeria Genome Sequencing Consortium, http://www.blugen.org/) with blastn [27] and subsequent manual inspection using dotplot sequence comparison (DOTTER, [56]). Satine represents the consensus of all the identified Bgh copies based on Clustalw [57] alignments.

\section{TE de novo detection and annotation in Bgh genome}

The TEdenovo pipeline $[19,20]$ from the REPET package [https://urgi.versailles.inra.fr/Tools/REPET] was launched on the BluGen Bgh assembly of isolate DH14 (BluGen, Blumeria Genome Sequencing Consortium, http:// www.blugen.org/) to detect and classify 2251 TE consensus sequences. The TEdenovo pipeline detects TE copies, groups them into families and defines the consensus sequence for each family containing at least three copies. These TE consensus sequences are classified according to their structural features and similarities with known TEs. In addition to Repbase update v16.03 [26] used at the TEdenovo pipeline similarity search step to classify TE consensus sequences, the Bgt_RepeatLibrary TE library [22] and a Bgh_TE library (unpublished) including Satine and two already characterized Bgh class I - SINE retroelements (Bgh_EGR1_cons and Bgh_EGH24_cons) [24, 25] were included. A first TEannot pipeline [21] from the REPET package was launched to annotate TE copies in the genome. A copy is considered as a full-length copy if the assembly of all its fragments represents 95 to $105 \%$ of the TE consensus length. This first TEannot pipeline found full-length copies for at least 1465 consensus sequences (hereafter named Blgr_refTEs_FL library) that were used to launch a second TEannot pipeline. In order to refine TE annotation, Blgr_refTEs_FL was manually curated by curating or deleting potential chimeric consensus sequences and consensus sequences shorter than $0.4 \mathrm{~Kb}$. A library of 733 TE consensus sequences (hereafter named Blgr_refTEs) was finally obtained, including Satine and the two already characterized Bgh class I - SINE retroelements Eg-R1_cons and Egh24 [24, 25], and was used to annotate the genome TE content. The TE consensus sequences (Fasta) and Annotation file (GFF) are available at: https://urgi.versailles.inra.fr/download/fungi/TEs/ (http:// doi.org/10.15454/1.4454357532232493E12 and http:// doi.org/10.15454/1.44543859671938E12).

\section{Search for the full-length LINE retrotransposon containing Avrk1 in ORF1}

Using Blgr_refTEs_FL genome annotation, we extracted all the full-length fragments (alignment length between 95 and $105 \%$ of the TE consensus length) corresponding to the seven TE consensus sequences that were homologous to full-length Avrk1 from Bgh isolate CC148 [4] at the expected location of an ORF1 of a LINE retrotransposon, together with 3000 bp flanking sequence on each side. We obtained 34 extended TE genome fragments that we annotated using PASTEC TEclassifier [20].

\section{Search for EKA TE families}

We searched for EKA TE families using AVRA10 (gi|111035036|gb|DQ679913| Blumeria graminis f. sp. hordei isolate CC148 Avra10 mRNA, complete CDS) and AVRK1 (gi|111035034|gb|DQ679912.1| Blumeria graminis f. sp. hordei isolate CC148 complete CDS) protein sequences as query for a blastx search [27] (e-value threshold 1e-05) in the Blgr_refTEs TE consensus library. We divided the results into three categories: two containing TE consensus sequences very similar to and AVRK1 and AVRA10 respectively (e-value $<1 \mathrm{e}-15$ ), and a third containing TE consensus sequences equidistant from AVRK1 and AVRA10.

\section{Plot Satine-like and Kryze-like copies on TE consensus}

We used PlotCovergage.py program from the S-MART package [58] to plot the coordinates of Satine-like and Kryze-like copies on their respective TE consensus sequences.

\section{Search for AVR/effector candidates}

We used blast (tblastn) [27] to search AVRK1 and AVRA10 peptide sequences against in-house databases of copies of TE consensus sequences similar to AVRK1 and AVRA10 ( $\mathrm{e}<=1 \mathrm{e}-05$, length of hit $>50$ amino acids). We discarded the shorter sequences (length $<75 \%$ of AVRK1/AVRA10 length) and aligned the remaining sequences and AVRK1 or AVRA10 using muscle [59], identified the 63 amino 
acids that represent the AVRK1/AVRA10 core sequence [4] and discarded the aligned sequences with a shorter core sequence ( $<75 \%$ AVRK1/AVRA10 core). We also discarded the sequences with a stop codon in the region aligned with AVRK1 or AVRA10. The resulting sequences were used to compile a database of predicted 415 EKA proteins (Table 3). We identified the NB domain in the remaining sequences using fuzzpro [60] and classified the sequences as full-length or truncated depending on the position of the stop codons.

\section{Phylogenetic analyses}

We combined the protein sequences from both AVRK1 and AVRA10 analyses, aligned them with muscle [59] and edited the alignment using jalview [61]. We selected the sequences that were at least $90 \%$ of the length of AVRK1 and removed 27 sequences that were redundant in that region, resulting in 317 sequences for the phylogenetic analyses. We used the BEAUti/BEAST software package v. 1.8.1 [62] to infer Bayesian phylogeny (the subsequent cited programs are also distributed with BEAST). We carried out two independent runs of 100,000,000 generations with sampling every 1000 generations. The runs were combined using Tracer v. 1.5.0. The Effective Sample Size (ESS) for the posterior distribution, likelihood and treeLikelihood showed satisfactory values, showing that the MCMC chain has been run for long enough to get a valid estimate of the parameter. The trees from both runs were combined using LogCombiner. In order to limit the analysis to the part of the trace that is in equilibrium, we discarded the initial 10,000,000 runs (burn-in value equal to $10 \%$ ). We generated a summary tree using TreeAnnotator and visualized it with Figtree. The sequence alignment used to produce the phylogeny is available at LabArchives. http://doi.org/10.6070/H4X34VG0.

\section{Positive selection analysis}

We used the program codeml from the package PAML 4.8 [63] to detect positive selection that may affect some sites in either AVRK1-like or AVRA10-like group of sequences. We used six different codon-based likelihood models (M0, M1a, M2a, M3, M7 and M8) to detect positively selected amino acid sites. The models are implemented in a maximum likelihood framework and tested using a likelihood ratio test (LRT).

\section{Search for protein structural domains}

We used Batch Web CD-search tool [64], CDART tool [65], HMM Superfamily search [66] and HHpred [67] to look for known structural domains.

\section{EKA protein identification}

The database of 415 predicted EKA proteins including AVRK1 and AVRA10 (Table 3) was merged with the Bgh
DH14 protein database on the Blugen website (http:// www.blugen.org/) in order to have a database search space that better reflects a complete proteome. The resulting database (of 6,885 sequences and 3,289,563 amino-acid residues) was used to query the mass spectrometry (MS) data sets from a large-scale analysis of the haustorial and hyphal proteomes of Bgh DH14 [44]. The original MS and Mascot data as well as associated metadata are publicly available and can be retrieved from the PRIDE database (Accession Numbers 1591715924; http://www.ebi.ac.uk/pride/). The search parameters were the same as in [44], but using Mascot vs. 2.4 (MatrixScience, London) as search engine. A first survey Mascot search was performed by including a "tolerant search" to allow any post-transcriptional modifications to be detected. From this search it was seen that most of the peptides identified were rich in proline $(\mathrm{P})$ and many were detected as hydroxyprolines. A subsequent search was then performed adding oxidised $\mathrm{P}$ in the variable modification. Proteins were validated by the identification of at least two significant peptides, with a Mascot score above 20 and above the identity threshold $(p<0.05)$.

\section{Availability of supporting data}

The data sets supporting the results of this article are available in:

INRA. http://doi.org/10.15454/1.4454357532232493E12. TE consensus library generated from Bgh genome assembly.

INRA. http://doi.org/10.15454/1.44543859671938E12. Bgh genome: TEs annotation file.

LabArchives. http://doi.org/10.6070/H4X34VG0. Sequence alignment used to produce the phylogeny.

PRIDE database (Accession Numbers 15917-15924; http://www.ebi.ac.uk/pride/). Original MS and Mascot data and associated metadata for protein identification.

\section{Additional files}

Additional file 1: Figure S1. Alignment of nucleotide sequences of Avra10 gene and Satine ORF1. (PDF $131 \mathrm{~kb}$ )

Additional file 2: Table S1. Mass spectometric data results for proteomic analysis using the database of predicted EKA proteins merged with the Bgh DH14 protein database. (XLSX $33 \mathrm{~kb}$ )

Additional file 3: Table S2. Peptides present en each protein accession. (XLSX $21 \mathrm{~kb})$

\section{Abbreviations}

AVR: Avirulence; Bgh: Blumeria graminis f.sp. hordei; Bgt: B. graminis f.sp. tritici; BluGen: Blumeria Genome Sequencing Consortium; CID: Collision induced dissociation; EKA: Effectors homologous to Avrk1 and Avra10; ESS: Effective Sample Size; ETI: Effector-triggered immunity; ḦIGS: Host-induced gene silencing; LRT: Likelihood Ratio Test; MS: Mass spectrometry; NB: Nucleotide binding; ORF: Open reading frame; ORF1p: ORF1 protein; R: Resistance; RIP: Repeat-induced point mutation; RH: RNase H; RRM: RNA-recognition motif; RT: Reverse transcriptase; TE: Transposable Element. 


\section{Competing Interests}

The authors declare that they have no competing interests.

\section{Authors' contributions}

JA carried out the analyses for TE de novo detection and annotation in Bgh genome, the search for the EKA TE families and the full-length LINE retrotransposon containing Avrk1 and the plot of Satine-like and Kryze-like copies on TE consensus and participated in the design of the study and in writing the manuscript. MV carried out the search for AVR/effector candidates and the phylogenetic and positive selection analyses and participated in the design of the study and in writing the manuscript. SO and TW built the consensus Satine model and participated in the conception and design of the study and in writing the manuscript. JKMB and PDS participated in the conception and design of the study and in writing the manuscript. LVB did the proteomic analysis and participated in writing the manuscript. PS did the search of protein structural domains and revised the manuscript. HQ participated in the design of the study and in writing the manuscript. SS coordinated the study, participated in its conception and design, and wrote the manuscript. All authors read and approved the final manuscript

\section{Acknowledgements}

JKMB is supported by the BBSRC Biotic Interactions Institute Strategic Programme. SS is supported by grant BIO2012- 32910 of Ministerio de Economía y Competitividad of the Spanish Government.

\section{Author details}

'INRA, UR1164 URGI Unité de Recherche Génomique-Info, Institut National de la Recherche Agronomique de Versailles-Grignon, Versailles 78026, France. ${ }^{2}$ INRA, UR1290 BIOGER, Biologie et gestion des risques en agriculture, Campus AgroParisTech, 78850 Thiverval-Grignon, France. ${ }^{3}$ John Innes Centre, Norwich Research Park, Norwich NR4 7UH, UK. Institute of Plant Biology, University of Zurich, Zollikerstrasse 107, 8008 Zurich, Switzerland. ${ }^{5}$ School of Biological Sciences, Royal Holloway University of London, Egham TW20 OEX, UK. ' Laboratory of Genetics, Department of Biotechnology, Agricultural University of Athens, lera Odos 75, TK 11855 Athens, Greece. 7 Department of Life Sciences, Imperial College London, London, UK. ${ }^{8}$ Centro de Biotecnología y Genómica de Plantas (UPM-INIA) and E.T.S.I. Agrónomos, Universidad Politécnica de Madrid, Campus de Montegancedo, Pozuelo de Alarcón 28223Madrid, Spain.

Received: 25 July 2015 Accepted: 3 November 2015 Published online: 10 November 2015

\section{References}

1. Ma W, Guttman DS. Evolution of prokaryotic and eukaryotic virulence effectors. Curr Opin Plant Biol. 2008;11(4):412-9.

2. Jones J, Dangl J. The plant immune system. Nature. 2006;444(7117):323-9.

3. Sacristan S, Garcia AF. The evolution of virulence and pathogenicity in plant pathogen populations. Mol Plant Pathol. 2008;9(3):369-84

4. Ridout CJ, Skamnioti P, Porritt O, Sacristan S, Jones JDG, Brown JKM. Multiple avirulence paralogues in cereal powdery mildew fungi may contribute to parasite fitness and defeat of plant resistance. Plant Cell. 2006;18(9):2402-14.

5. Nowara D, Gay A, Lacomme C, Shaw J, Ridout C, Douchkov D, et al. HIGS. Host-Induced Gene Silencing in the Obligate Biotrophic Fungal Pathogen Blumeria graminis. Plant Cell. 2010;22(9):3130-41.

6. Shen Q, Saijo Y, Mauch S, Biskup C, Bieri S, Keller B, et al. Nuclear activity of MLA immune receptors links isolate-specific and basal disease-resistance responses. Science. 2007:315(5815):1098-103.

7. Spanu P, Abbott J, Amselem J, Burgis T, Soanes D, et al. Genome Expansion and Gene Loss in Powdery Mildew Fungi Reveal Tradeoffs in Extreme Parasitism. Science. 2010;330(6010):1543-6.

8. Kusch S, Ahmadinejad N, Panstruga R, Kuhn H. In silico analysis of the core signaling proteome from the barley powdery mildew pathogen (Blumeria graminis f.sp hordei). BMC Genomics. 2014;15:843.

9. Sacristan S, Vigouroux M, Pedersen C, Skamnioti P, Thordal-Christensen $H_{\text {, }}$ Micali C, et al. Coevolution between a Family of Parasite Virulence Effectors and a Class of LINE-1 Retrotransposons. PLoS One. 2009:4(10):e7463.

10. Jones L, Riaz S, Morales-Cruz A, Amrine KCH, McGuire B, Gubler WD, et al. Adaptive genomic structural variation in the grape powdery mildew pathogen, Erysiphe necator. BMC Genomics. 2014;15:1081.
11. Tollenaere C, Susi H, Nokso-Koivisto J, Koskinen P, Tack A, Auvinen P, et al. SNP Design from 454 Sequencing of Podosphaera plantaginis Transcriptome Reveals a Genetically Diverse Pathogen Metapopulation with High Levels of Mixed-Genotype Infection. PLoS One. 2012;7(12):e52492.

12. Wicker T, Sabot F, Hua-Van A, Bennetzen JL, Capy P, Chalhoub B, et al. A unified classification system for eukaryotic transposable elements. Nat Rev Genet. 2007:8(12):973-82.

13. Eickbush TH, Malik HS. Origins and evolution of retrotransposons. In: Craig NL, Craigie R, Gellert M, Lambowitz AM, editors. Mobile DNA II. Washington DC: American Society of Microbiology Press; 2002. p. 1111.

14. Kapitonov W, Tempel S, Jurka J. Simple and fast classification of non-LTR retrotransposons based on phylogeny of their RT domain protein sequences. Gene. 2009:448(2):207-13.

15. Novikova OS, Blinov AG. Origin, evolution, and distribution of different groups of non-LTR retrotransposons among eukaryotes. Genetika. 2009;45(2):149-59.

16. Sokolowski M, De Haro D, Christian CM, Kines KJ, Belancio VP. Characterization of L1 ORF1p Self-Interaction and Cellular Localization Using a Mammalian Two-Hybrid System. PLoS One. 2013;8:12.

17. Martin SL. Nucleic acid chaperone properties of ORF1p from the non-LTR retrotransposon, LINE-1. RNA Biol. 2010;7(6):706-11.

18. Khazina $E$, Weichenrieder $O$. Non-LTR retrotransposons encode noncanonical RRM domains in their first open reading frame. Proc Natl Acad Sci U S A. 2009;106(3):731-6.

19. Flutre T, Duprat E, Feuillet C, Quesneville H. Considering Transposable Element Diversification in De Novo Annotation Approaches. PLoS One. 2011:6(1):e16526.

20. Hoede C, Arnoux S, Moisset M, Chaumier T, Inizan O, Jamilloux V, et al. PASTEC: An Automatic Transposable Element Classification Tool. PLoS One. 2014;9(5):e91929.

21. Quesneville H, Bergman C, Andrieu O, Autard D, Nouaud D, Ashburner M, et al. Combined evidence annotation of transposable elements in genome sequences. PLoS Comput Biol. 2005;1(2):166-75.

22. Parlange F, Oberhaensli S, Breen J, Platzer M, Taudien S, Simkova H, et al. A major invasion of transposable elements accounts for the large size of the Blumeria graminis f.sp tritici genome. Funct Integr Genomics. 2011;11(4):671-7.

23. He C, Nourse J, Kelemu S, Irwin J, Manners J. CgT1: A non-LTR retrotransposon with restricted distribution in the fungal phytopathogen Colletotrichum gloeosporioides. Mol Gen Genet. 1996;252(3):320-31.

24. Rasmussen M, Rossen L, Giese H. Sine-Like Properties of a Highly Repetitive Element in the Genome of the Obligate Parasitic Fungus Erysiphe-graminis f.sp hordei. Mol Gen Genet. 1993;239(1-2):298-303.

25. Wei YD, Collinge DB, Smedegaard-Petersen V, Thordal-Christensen H. Characterization of the transcript of a new class of retroposon-type repetitive element cloned from the powdery mildew fungus, Erysiphe graminis. Mol Gen Genet. 1996;250(4):477-82

26. Jurka J, Kapitonov V, Pavlicek A, Klonowski P, Kohany O, Walichiewicz J. Repbase update, a database of eukaryotic repetitive elements. Cytogenet Genome Res. 2005;110(1-4):462-7.

27. Altschul S, Gish W, Miller W, Myers E, Lipman D. Basic Local Alignment Search Tool. J Mol Biol. 1990;215(3):403-10.

28. Dong N-P, Zhang L-X, Liang Y-Z. A comprehensive investigation of proline fragmentation behavior in low-energy collision-induced dissociation peptide mass spectra. Int J Mass Spectrom. 2011;308:89-97.

29. Wicker T, Oberhaensli S, Parlange F, Buchmann JP, Shatalina M, Roffler S, et al. The wheat powdery mildew genome shows the unique evolution of an obligate biotroph. Nat Genet. 2013;45(9):1092-8.

30. Amselem J, Lebrun M, Quesneville $H$. Whole genome comparative analysis of transposable elements provides new insight into mechanisms of their inactivation in fungal genomes. BMC Genomics. 2015;16:141.

31. Pedersen C, van Themaat EVL, McGuffin LJ, Abbott JC, Burgis TA, Barton G, et al. Structure and evolution of barley powdery mildew effector candidates. BMC Genomics. 2012;13:694.

32. Raffaele S, Kamoun S. Genome evolution in filamentous plant pathogens: why bigger can be better. Nat Rev Microbiol. 2012;10(6):417-30.

33. Spanu PD. The Genomics of Obligate (and Nonobligate) Biotrophs. Annu Rev Phytopathol. 2012;50:91-109.

34. Troch V, Audenaert K, Wyand RA, Haesaert G, Hofte M, Brown JKM. Formae speciales of cereal powdery mildew: close or distant relatives? Mol Plant Pathol. 2014;15(3):304-14 
35. Brosius J, Gould SJ. On "genomenclature": a comprehensive (and respectful) taxonomy for pseudogenes and other "junk DNA". Proc Natl Acad Sci U S A. 1992:89:10706.

36. Chenais B, Caruso A, Hiard S, Casse N. The impact of transposable elements on eukaryotic genomes: From genome size increase to genetic adaptation to stressful environments. Gene. 2012;509(1):7-15.

37. de Souza FSJ, Franchini LF, Rubinstein M. Exaptation of Transposable Elements into Novel Cis-Regulatory Elements: Is the Evidence Always Strong? Mol Biol Evol. 2013;30(6):1239-51.

38. Alzohairy AM, Gyulai G, Jansen RK, Bahieldin A. Transposable elements domesticated and neofunctionalized by eukaryotic genomes. Plasmid. 2013;69(1):1-15.

39. Kapitonov $V$, Jurka J. RAG1 core and $V(D) J$ recombination signal sequences were derived from Transib transposons. PLoS Biol. 2005;3(6):998-1011.

40. Lavialle C, Cornelis G, Dupressoir A, Esnault C, Heidmann O, Vernochet C, et al. Paleovirology of 'syncytins', retroviral env genes exapted for a role in placentation. Philos Trans R Soc B-Biol Sci. 2013;368(1626):20120507.

41. Pardue M, DeBaryshe P. Retrotransposons provide an evolutionarily robust non-telomerase mechanism to maintain telomeres. Annu Rev Genet. 2003:37:485-511.

42. Pardue M, DeBaryshe PG. Retrotransposons that maintain chromosome ends. Proc Natl Acad Sci U S A. 2011;108(51):20317-24.

43. Brown JKM, Tellier A. Plant-Parasite Coevolution: Bridging the Gap between Genetics and Ecology. Annu Rev Phytopathol. 2011;49:345-67.

44. Bindschedler LV, McGuffin LJ, Burgis TA, Spanu PD, Cramer R. Proteogenomics and in silico structural and functional annotation of the barley powdery mildew Blumeria graminis f. sp. hordei. Methods. 2011;54:432-41.

45. Lo Presti L, Lanver D, Schweizer G, Tanaka S, Liang L, Tollot M, et al. Fungal Effectors and Plant Susceptibility. Annu Rev Plant Biol. 2015;66:513-45.

46. Perfect SE, O'Connell RJ, Green EF, Doering-Saad C, Green JR. Expression cloning of a fungal proline-rich glycoprotein specific to the biotrophic interface formed in the Colletotrichum-bean interaction. Plant J. 1998;15:273-9.

47. Takahara H, Dolf A, Endl E, O'Connell R. Flow cytometric purification of Colletotrichum higginsianum biotrophic hyphae from Arabidopsis leaves for stagespecific transcriptome analysis. Plant J. 2009;59:672-83.

48. Bolton MD, van Esse HP, Vossen JH, Jonge R, Stergiopoulos I, Stulemeijer IJE, et al. The novel Cladosporium fulvum lysin motif effector Ecp6 is a virulence factor with orthologues in other fungal species. Mol Microbiol. 2008;69(1):119-36.

49. Kleemann J, Rincon-Rivera L, Takahara H, Neumann U, van Themaat EVLH, van der Does C, et al. Sequential Delivery of Host-Induced Virulence Effectors by Appressoria and Intracellular Hyphae of the Phytopathogen Colletotrichum higginsianum. PLoS Pathog. 2012;8(4):e1002643.

50. Krishna S, Majumdar I, Grishin N. Structural classification of zinc fingers Nucleic Acids Res. 2003;31(2):532-50.

51. Okada N, Sasaki T, Shimogori T, Nishihara H. Emergence of mammals by emergency: exaptation. Genes Cells. 2010;15(8):801-12.

52. Miller W, Capy P. Mobile genetic elements as natural tools for genome evolution, Mobile Genetic Elements. Protocols and Genomic Applications. 2004;260:1-20.

53. Thomas CM, Macias F, Alonso C, Lopez MC. The biology and evolution of transposable elements in parasites. Trends Parasitol. 2010;26(7):350-62.

54. Kasuga T, Gijzen M. Epigenetics and the evolution of virulence. Trends Microbiol. 2013;21(11):575-82

55. Weiberg A, Wang M, Lin F, Zhao H, Zhang Z, Kaloshian I, et al. Fungal Small RNAs Suppress Plant Immunity by Hijacking Host RNA Interference Pathways. Science. 2013;342(6154):118-23.

56. Sonnhammer ELL, Durbin R. A Dot-Matrix Program with Dynamic Threshold Control Suited for Genomic DNA and Protein-Sequence Analysis. Gene-Combis. 1995;167:1

57. Thompson J, Gibson T, Higgins D. Multiple sequence alignment using ClustalW and ClustalX. Curr Protoc Bioinformatics. 2002; Chapter 2:Unit 2.3.

58. Zytnicki M, Quesneville H. S-MART, A Software Toolbox to Aid RNA-seq Data Analysis. PLoS One. 2011;6(10):e25988.

59. Edgar R. MUSCLE: multiple sequence alignment with high accuracy and high throughput. Nucleic Acids Res. 2004;32(5):1792-7.

60. Rice P, Longden I, Bleasby A. EMBOSS: The European molecular biology open software suite. Trends Genet. 2000;16(6):276-7.

61. Waterhouse AM, Procter JB, Martin DMA, Clamp M, Barton GJ. Jalview Version 2-a multiple sequence alignment editor and analysis workbench. Bioinformatics. 2009;25(9):1189-91.
62. Drummond AJ, Suchard MA, Xie D, Rambaut A. Bayesian Phylogenetics with BEAUti and the BEAST 1.7. Mol Biol Evol. 2012;29(8):1969-73.

63. Yang Z. PAML 4: Phylogenetic analysis by maximum likelihood. Mol Biol Evol. 2007;24(8):1586-91.

64. Marchler-Bauer A, Lu S, Anderson JB, Chitsaz F, Derbyshire MK, DeWeese-Scott C, et al. CDD: a Conserved Domain Database for the functional annotation of proteins. Nucleic Acids Res. 2011;39:D225-9.

65. Geer LY, Domrachev M, Lipman DJ, Bryant SH. CDART: Protein homology by domain architecture. Genome Res. 2002;12(10):1619-23.

66. Gough J, Karplus K, Hughey R, Chothia C. Assignment of homology to genome sequences using a library of hidden Markov models that represent all proteins of known structure. J Mol Biol. 2001;313(4):903-19.

67. Soding J. Protein homology detection by HMM-HMM comparison. Bioinformatics. 2005;21(7):951-60.

\section{Submit your next manuscript to BioMed Central and take full advantage of:}

- Convenient online submission

- Thorough peer review

- No space constraints or color figure charges

- Immediate publication on acceptance

- Inclusion in PubMed, CAS, Scopus and Google Scholar

- Research which is freely available for redistribution 